\title{
SECOND EIGENMODE OPERATION OF SUSPENDED MICROCHANNEL RESONATORS FOR HIGH PRECISION FLOW-THROUGH MASS SENSING
}

\author{
J. Lee and S.R. Manalis*
}

Department of Biological Engineering, Massachusetts Institute of Technology, Massachusetts, USA

\section{ABSTRACT}

An intrinsic uncertainty in particle mass sensing with the suspended microchannel resonator (SMR) results from variation in a particle's position near the free end of the resonator. To circumvent this error without modifying the resonator geometry, we employ the second flexural bending mode of the SMR which exhibits additional frequency shift peaks while particles pass over antinodes. The antinode peaks have the attractive property that they are insensitive to the lateral position of the particle within the microchannel. We measure polystyrene beads with the first and second modes. For $3 \mu \mathrm{m}$ diameter beads, the second mode sensing at antinodes improves the coefficient of variation (CV) in buoyant mass from 1.76 to $1.05 \%$ for population measurements and from 1.40 to $0.53 \%$ for a single particle trapped.

\section{INTRODUCTION}

Although the SMR has a $\sim 1$ femtogram resolution in a $1 \mathrm{~Hz}$ bandwidth and can weigh single nanoparticles and cells as they transit the embedded microchannel [1], the particle flow path near the tip of the SMR is uncertain (Fig. 1(a)) and this variation can reduce precision of the mass measurement. This position dependent error can broaden the mass histogram of a population of particles and introduce uncertainty during single particle trapping where the mass of single particle is measured repeatedly [2]. Employing doubly clamped resonators, hydrodynamic particle focusing, or particle trapping at predefined mechanical structures could reduce this measurement uncertainty. However these strategies either require fabricating new SMR structures and fluidic interfaces or may reduce the measurement throughput. In this work, we show that the position dependent error can be eliminated in existing SMR structures without sacrificing throughput by utilizing higher order flexural bending modes.

\section{THEORY}

When a point mass is deposited on a vibrating cantilever, the resulting change in resonant frequency depends strongly on the location of the added mass. From the Euler-Bernoulli beam equation, displacement at a position normalized by the cantilever length is given by:

$$
\begin{gathered}
u_{n}\left(\frac{x}{L}\right)=\frac{A_{n}}{2}\left[\left(\cosh \left(\frac{\lambda_{n} x}{L}\right)-\cos \left(\frac{\lambda_{n} x}{L}\right)\right)-B\left(\lambda_{n}\right)\left(\sinh \left(\frac{\lambda_{n} x}{L}\right)-\sin \left(\frac{\lambda_{n} x}{L}\right)\right)\right] \\
B\left(\lambda_{n}\right)=\left(\cosh \left(\lambda_{n}\right)+\cos \left(\lambda_{n}\right)\right) /\left(\sinh \left(\lambda_{n}\right)+\sin \left(\lambda_{n}\right)\right)
\end{gathered}
$$

where $x$ is the position from the clamped base, $L$ is the cantilever length, $A_{n}$ is the tip amplitude, and $\lambda_{n}$ is the eigenvalue for the $n^{\text {th }}$ flexural bending modes; 1.8751, 4.6941, 7.8548, and 10.996 are eigenvalues for the first four bending modes, respectively [3]. Using this mode shape shown in Fig. 1(b) (top) and applying the Rayleigh-Ritz theorem which equates the time averaged kinetic energy and strain energy at resonance, the relative resonance frequency shift of the cantilever is estimated by

$$
(\Delta f / f)_{n}=-1+\left(1+u_{n}(x / L)^{2} \cdot \Delta m / m_{e f f}\right)^{-0.5}
$$

where $A_{n}=1$ (normalized mode shape), $f$ is the resonance frequency, and $m_{\text {eff }}$ is the effective mass of the cantilever when there is no particle [4]. Figure 1(b) shows mode shapes and relative resonance frequency shifts for the first four flexural bending modes when 2

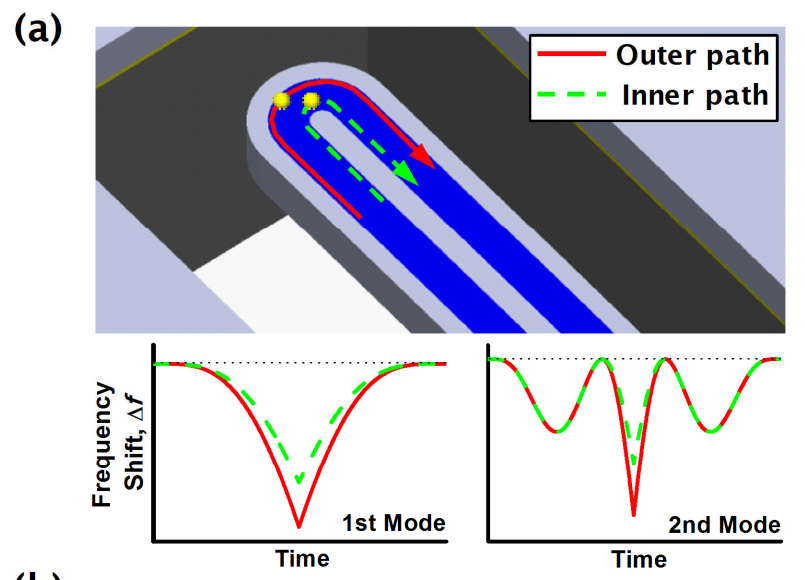

(b)

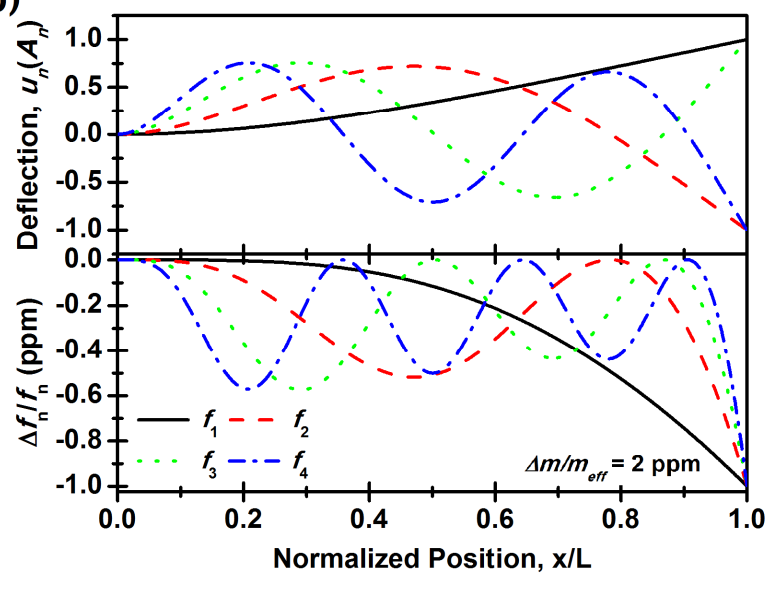

Figure 1: (a) A particle may transit anywhere between the inner and outer channel wall. A particle that makes a U-turn near the inner wall results in a smaller frequency shift than if it turns near the outer wall. (b) Mode shape and resonance frequency shift upon point mass loading on a cantilever as a function of the position of the added mass normalized by the cantilever length. For a cantilever that resonates at its $n^{\text {th }}(n>=1)$ flexural bending mode, there are $2 n-1$ peaks in the frequency shift measured during a single particle transit (one peak at the free end and $2 n-2$ peaks at antinodes).

parts-per-million (ppm) of mass is added at various positions along the cantilever.

When a particle transits the U-shaped embedded microchannel of the SMR, there are $2 n-1$ frequency shift peaks at the $n^{\text {th }}$ flexural bending mode. As shown in Fig. 1(a), a particle flowing through the microchannel can travel along random paths. In the most extreme cases, the particle can be positioned at the inner and outer edges of the microchannel. Since $\Delta m / m_{\text {eff }}$ in equation (2) is much less than unity, the maximum position-dependent error is estimated by

$$
\left(\frac{f_{i}-f_{o}}{f-f_{m}}\right) \approx \frac{u_{n}\left(x_{i} / L\right)^{2}-u_{n}\left(x_{o} / L\right)^{2}}{u_{n}\left(x_{m} / L\right)^{2}}
$$


where, the subscripts $i, o$, and, $m$ denote particle positions at the inner edge, the outer edge, and the midpoint of the channel, respectively. The maximum position error is $\sim 11 \%$ for a $210 \mu \mathrm{m}$ long SMR used in first mode sensing. However, the frequency shift measured at antinodes is nearly insensitive to the lateral position of the particle in the channel (see Fig. 1(a) for comparison of the first and second mode sensing).

\section{RESULTS AND DISCUSSIONS}

To date, only the fundamental mode of the SMR has been utilized and higher vibration modes have yet to be explored. For microcantilevers, it has been shown that performance in a viscous medium can be improved by operating them at higher modes since their quality factors increase with mode number [5]. However this is not always the case for vacuum packaged SMRs since the mechanisms that give rise to viscous loss are different.

SMR devices used in this work are $406 \mu \mathrm{m}$ long, $28.5 \mu \mathrm{m}$ wide, and $12 \mu \mathrm{m}$ thick, and their channel cross-sections are $7.9 \times 8$ $\mu \mathrm{m}^{2}$. Figure 2 shows that resonance frequencies of the first, second, and third flexural bending modes decrease linearly with increasing fluid density as expected. Interestingly, the quality factor of each mode exhibits non-monotonic behaviors which decrease or increase with increasing viscosity (Fig. 3). The local minimum in the plot of quality factor vs. viscosity occurs at higher viscosities as the mode number rises. Observed non-monotonic trends may be explained with the theory for the first mode [6] but the effects of compressibility in the fluid become increasingly important for higher modes since the resonance frequency increases. Detailed theoretical investigation considering the effects of compressibility is currently underway. In addition, the quality factors of higher modes are significantly lower than that of the first mode when the

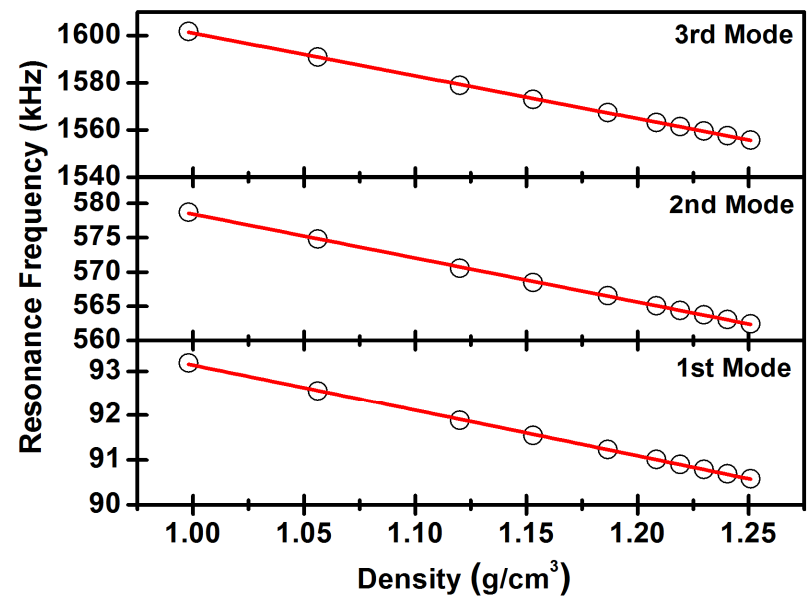

Figure 2: Resonance frequencies of the first, second, and third flexural bending modes are linear functions of fluid density. Density was varied by mixing glycerol with water in different ratios $(24 \sim 96 \%)$.

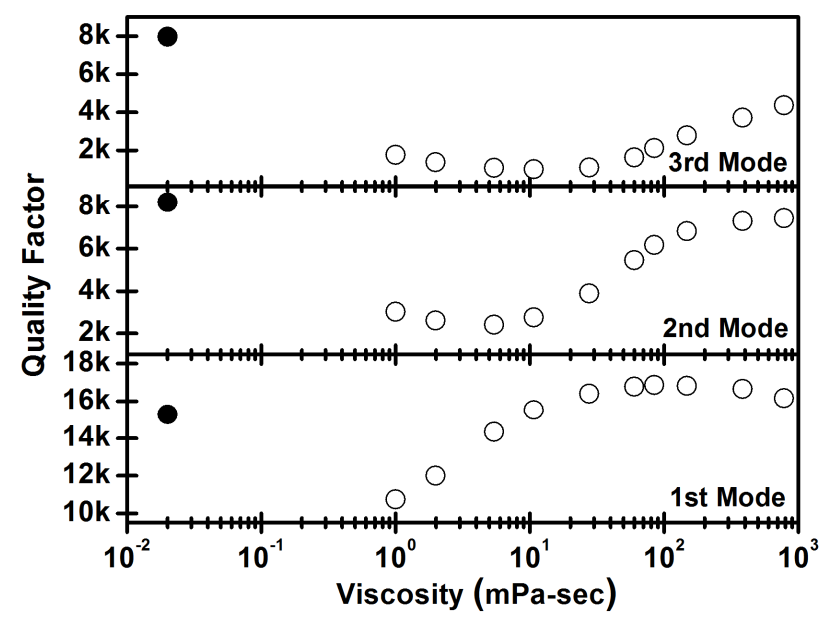

Figure 3: Quality factors of the first, second, and third flexural bending modes are highly non-monotonic functions of fluid viscosity. Viscosity was varied by mixing glycerol with water in different ratios $(24 \sim 96 \%)$. Solid circles represent quality factors of dry SMRs filled with air.

SMR is filled with water $\left(Q_{1}=10,742, Q_{2}=3,023\right.$, and $\left.Q_{3}=1,760\right)$. One potential drawback of higher mode sensing is that the reduced quality factor may result in a lower mass resolution. Thus, there is a tradeoff between mass resolution and precision that should be considered for a given application of the SMR.

We successfully operated the SMR in feedback mode with the first and second modes, however we found that feedback was not stable for higher order modes. This may be due to the increased stiffness as the mode number rises. In a $1 \mathrm{kHz}$ bandwidth, frequency noises measured were 0.439 and $0.142 \mathrm{ppm}$ for the first and second modes, respectively. While SMRs used in this work exhibit lower frequency noise with the second mode than with the first mode, this is not a universal characteristic. Three out of five different SMR designs allow feedback operation in both the first and second modes however only two out of the three designs exhibit better mass resolution with the second mode (Table 1). This indicates that there are preferable designs for higher mode operations and that the structures used in this work may not be optimal.

Monodispersed particles would be ideal for evaluating the performance of $2^{\text {nd }}$ mode sensing since dispersity may overshadow the position-dependent error. Typical CVs of commercially available NIST size standard beads are $1 \sim 2 \%$ in diameter $(3 \sim 6 \%$ in volume or mass) which are comparable to the position-dependent error. We therefore used a dynamic single particle trapping method where alternating pressure between bypass channels maintains a single particle in the suspended microchannel for repeated measurements [2]. Figure 4 shows resonance frequency shifts of the first and second modes as a nominally $4.17 \mu \mathrm{m}$ diameter

Table 1: Dimensions (length (l), width (w), and thickness (t)) and resonant characteristics of 5 existing SMRs. Resonance frequencies (f), quality factors $(Q)$, and mass resolutions $(\delta m)$ are measured with each SMR filled with water.

\begin{tabular}{|c|c|c|c|c|c|c|c|c|c|c|c|c|}
\hline$\#$ & $l(\mu \mathrm{m})$ & $w(\mu \mathrm{m})$ & $t(\mu \mathrm{m})$ & $f_{l}(\mathrm{kHz})$ & $Q_{1}$ & $f_{2}(\mathrm{kHz})$ & $Q_{2}$ & $\begin{array}{c}\delta m_{1, l \mathrm{kHz}} \\
(\mathrm{fg})\end{array}$ & $\begin{array}{c}\delta m_{1, l \mathrm{~Hz}} \\
(\mathrm{fg})\end{array}$ & $\begin{array}{c}\delta m_{2 a, l \mathrm{kHz}} \\
(\mathrm{fg})\end{array}$ & $\begin{array}{c}\delta m_{2 a, l \mathrm{~Hz}} \\
(\mathrm{fg})\end{array}$ & $\begin{array}{c}f_{2} \\
\mathrm{feedback}\end{array}$ \\
\hline 1 & 210 & 33 & 7 & 209.9 & 12,064 & 1297.5 & 4,798 & 10.2 & 0.24 & 37.7 & 0.29 & $\mathrm{O}$ \\
\hline 2 & 210 & 33 & 12 & 384 & 5,880 & 2311 & 1,760 & 14.1 & 0.71 & - & - & $\mathrm{X}$ \\
\hline 3 & 321 & 57 & 19 & 231 & 2,270 & 1396 & 810 & 113.7 & 4.6 & - & - & $\mathrm{X}$ \\
\hline 4 & 406 & 28.5 & 7 & 54.7 & 23,447 & 340.2 & 9,080 & 90.8 & 6.5 & 30.5 & 2.0 & $\mathrm{O}$ \\
\hline 5 & 406 & 28.5 & 12 & 93.2 & 10,742 & 578.7 & 3,023 & 48 & 0.7 & 35 & 5.4 & $\mathrm{O}$ \\
\hline
\end{tabular}




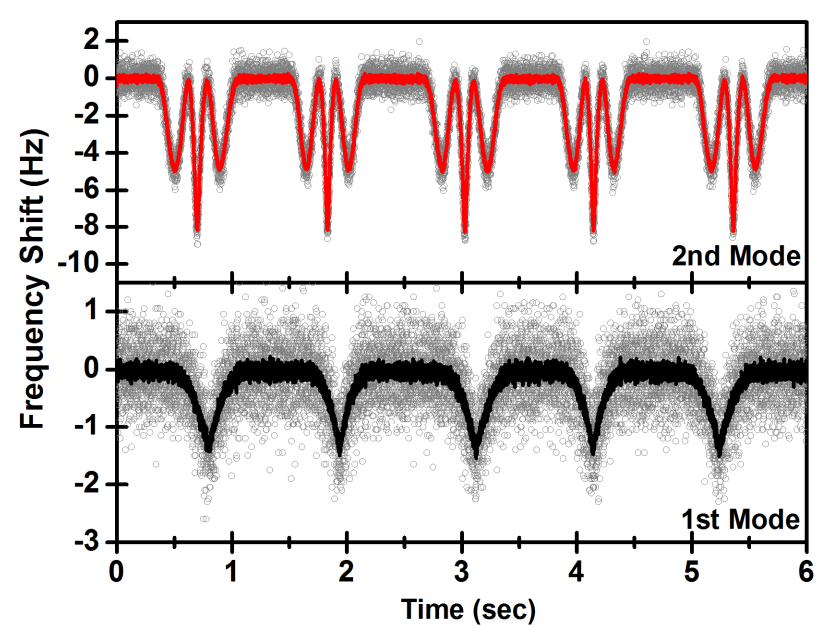

Figure 4: Frequency shift vs. time for a nominally $4.17 \mu \mathrm{m}$ diameter polystyrene bead that is repeatedly measured. Gray dots show raw data sampled at $2 \mathrm{kHz}$, and black (first mode) and red (second mode) solid lines show data smoothed with the Savitzky-Golay filter ( $n=12,3^{\text {rd }}$ order $)$.

polystyrene bead (NT21N, Bangs Laboratories) repeatedly transits the suspended microchannel. As expected from theory, the first mode sensing exhibits one peak near the tip and the second mode sensing exhibits three peaks per every trapping cycle.

Following calibration with $4.17 \mu \mathrm{m}$ diameter beads, we measured a population of $3 \mu \mathrm{m}$ diameter polystyrene beads (4203A, Thermo Scientific) and trapped a single particle drawn from the same population for about $1 \mathrm{hr}$. Figure 5 shows histograms of the relative frequency shift for the first mode tip, second mode tip, and second mode antinode sensing. For both population and trapping measurements, the second mode antinode sensing when the left and right antinode peaks are averaged offers the lowest $\mathrm{CV}\left(\Delta f_{2, a}(\operatorname{avg})\right)$.

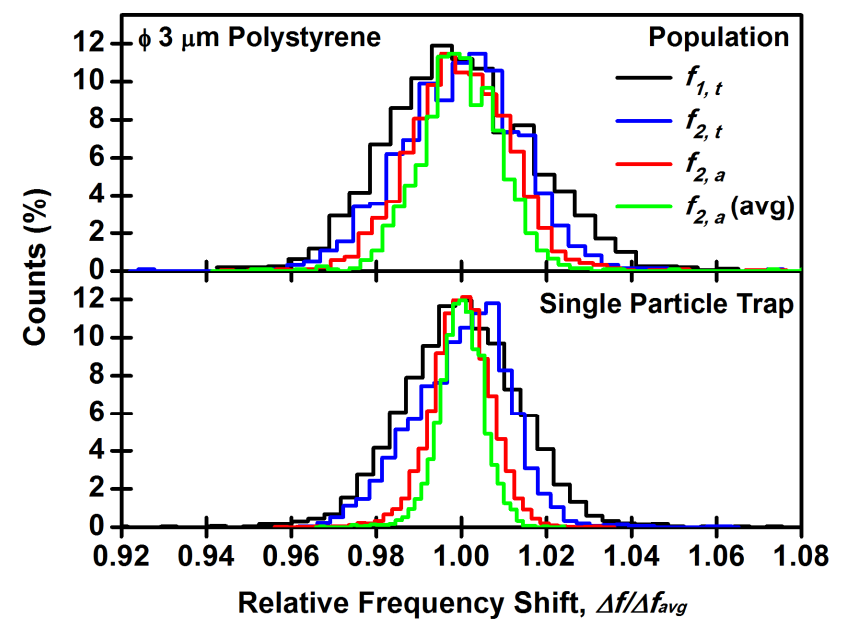

Figure 5: Histograms of relative frequency shifts for nominally 3 $\mu m$ diameter polystyrene particles (population) and repeated measurements of a particle drawn from the same population (single particle trapping). Subscripts 1 and 2 denote mode number and subscripts $t$ and a denote tip and antinode, respectively. The second mode sensing at antinodes generates the narrowest histogram for both population and trapping measurements when frequency shifts at the left and right antinodes are averaged.
Table 2: A summary of the first and second flexural mode sensing characteristics for population and trapping measurements with 3 $\mu m$ diameter polystyrene beads.

\begin{tabular}{|c|c|c|c|c|}
\hline \multicolumn{2}{|c|}{ Mode } & $\mu(\mathrm{Hz})$ & $\sigma(\mathrm{Hz})$ & $\mathrm{CV}(\%)$ \\
\hline \multirow{4}{*}{ Population } & $\Delta f_{l, t}$ & 0.590 & 0.010 & 1.76 \\
\cline { 2 - 5 } & $\Delta f_{2, t}$ & 3.258 & 0.047 & 1.45 \\
\cline { 2 - 5 } & $\Delta f_{2, a}$ & 1.962 & 0.024 & 1.24 \\
\cline { 2 - 5 } Trapping & $\Delta f_{2, a}(\mathrm{avg})$ & 1.962 & 0.021 & 1.05 \\
\hline & $\Delta f_{l, t}$ & 0.584 & 0.008 & 1.40 \\
\cline { 2 - 5 } & $\Delta f_{2, t}$ & 3.281 & 0.032 & 0.99 \\
\cline { 2 - 5 } & $\Delta f_{2, a}$ & 1.957 & 0.014 & 0.70 \\
\cline { 2 - 5 } & $\Delta f_{2, a}$ (avg) & 1.957 & 0.010 & 0.53 \\
\hline
\end{tabular}

Detailed results are summarized in Table 2. Interestingly, the second mode tip sensing also exhibits narrower dispersion than the first mode tip sensing for both population and single particle trapping although theory predicts more error with the second mode. The mass resolution in a $1 \mathrm{kHz}$ bandwidth is 48,35 , and $21 \mathrm{fg}$ for the first mode tip, second mode antinode, and second mode tip sensing, respectively.

\section{CONCLUSIONS}

This paper reports promising characteristics of the higher eigenmode operation of suspended microchannel resonators for flow-through mass sensing. Existing SMR devices can be operated at their second flexural bending modes to achieve high precision mass sensing and, in some cases, provide improved mass resolution.

\section{REFERENCES}

[1] T. P. Burg, M. Godin, S. M. Knudsen, W. Shen, G. Carlson, J. S. Foster, K. Babcock, and S. R. Manalis, "Weighing of biomolecules, single cells and single nanoparticles in fluid", Nature, 446, 1066 (2007).

[2] M. Godin, F. F. Delgado, S. Son, W. H. Grover, A. K. Bryan, A. Tzur, P. Jorgensen, K. Payer, A. D. Grossman, M. W. Kirschner, and S. R. Manalis, "Using buoyant mass to measure the growth of single cells", Nature Method, accepted for publication.

[3] A. N. Cleland, Foundations of Nanomechanics: from solid-state theory to device applications: Springer, 2003.

[4] S. Dohn, W. Svendsen, A. Boisen, and O. Hansen, "Mass and position determination of attached particles on cantilever based mass sensors", Review of Scientific Instruments, 78, 103303 (2007).

[5] T. Braun, M. K. Ghatkesar, N. Backmann, W. Grange, P. Boulanger, L. Letellier, H. Lang, A. Bietsch, C. Gerber, and M. Hegner, "Quantitative time-resolved measurement of membrane protein-ligand interactions using microcantilever array sensors", Nature Nanotechnology, 4, 179 (2008).

[6] T. P. Burg, J. E. Sader, and S. R. Manalis, "Nonmonotonic Energy Dissipation in Microfluidic Resonators", Physical Review Letters, 102, 228103 (2009).

\section{CONTACT}

*S.R. Manalis, tel: +1-617-253-0632; scottm@media.mit.edu 